\title{
Functional modeling applied to HAZOP automation
}

\author{
José Luis de la Mata Manuel Rodríguez
}

\begin{abstract}
In this paper we present a new tool to perform guided HAZOP analyses. This tool uses a functional model of the process that merges its functional and its structural information in a natural way. The functional modeling technique used is called D-higraphs. This tool solves some of the problems and drawbacks of other existing methodologies for the automation of HAZOPs. The applicability and easy understanding of the proposed methodology is shown in an industrial case.
\end{abstract}

Keywords: Functional modeling; HAZOP; Risk assessment.

\section{Introduction}

Currently, due to economical optimization of process plants, they are working at extreme conditions of pressure and temperature. This situation makes them more likely to fail and, even worse, the consequences of accidents are more severe. In addition, the Chemical Industry is facing tighter regulations and a growing attention of the media towards industrial accidents. Of course, we have also to consider the economical losses associated to accidents in terms of shutdowns, reparations, compensations and fines.

In the design stage of the process plant, Process Hazard Analyses (PHA) are carried out to identify potential sources of accidents and propose some solutions that enhance the safety of the plant. One of the most used of all of the existing PHA techniques is the Hazard and Operability Study (HAZOP) (Zhao et al., 2005). However, this approach consumes a lot of effort, time and, hence, money.

For these reasons, in the last two decades a lot of effort has been devoted to the implementation of tools and methodologies that can lead to the automation of these studies. In this work we present a new tool based on a functional modeling technique called D-higraphs. This approach takes into account in a natural way the structure as well as the functionality of the system (process and control system).

\section{D-higraphs: merging function and structure}

\subsection{Dualization: from Higraphs to D-higraphs}

Higraphs were first presented by Harel (1987) and they are a general kind of diagramming that can be understood as a combination and extension of Graphs, Euler/Venn diagrams and the Cartesian Product. They are well suited to specify complex concurrent systems but not process systems.

However, they can be adapted to process specification by the dualization of their properties and relations. This dualization has led to D-higraphs that were first presented in Rodríguez \& Sanz (2009). Higraphs and D-higraphs consist of blobs and edges connecting them.

It should be noted that the term 'dualization' is used in a different way than the one used in Dual-Graphs or Digraphs; it is only applied to the properties and not to the elements. 


\subsection{Elements of a D-higraph}

A basic blob and its elements are depicted in the left-hand-side of Fig. 1 and the different types of edges are shown in the center of Fig. 1. Blobs represent functions (transitions) that are performed by an ACTOR producing state/s 2 if the state/s 1 is enabled and if the condition is true. Edges represent flows of mass, energy, or information, which are responsible of all the interactions in a process system (Lind, 1994). Mass, energy and information edges are depicted differently, but the type of flow does not affect the behavior of the model, it is a just a visual aid.

Disjoint blobs imply an AND relation (both transitions between states take place) while orthogonal blobs represent an OR relation (only one of the transitions takes place). The main properties are:

- Blob connection. An edge always links two blobs. Under certain conditions, one of the blobs cannot be represented (elliptic blob), but it exists

- Blob inclusion. Blobs can be included inside of other blobs (Venn diagram inclusion). This means that the inner blob performs a function that is necessary for the function of the outer blob (representation of functions hierarchy).

- Blob partition. A blob can be partitioned into orthogonal components, establishing an OR condition between the partitions.

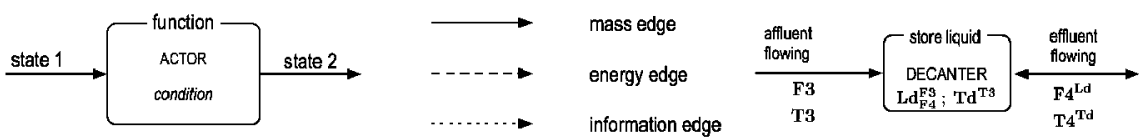

Fig. 1. Basic blob, types of edges, and three layer D-higraph.

\subsection{Causality and qualitative reasoning}

The main objective of D-higraphs is not only the representation of knowledge about process systems. De la Mata \& Rodríguez (2010a,b) provide a series of causation rules relating two events that allow us to track the evolution and propagation of failures across the system. This rules combined with sensor data of the plant enables the possibility of performing FDI analysis using D-higraph models.

However, certain analyses require the use of deviations and not only failures, like HAZOP studies. In a certain way, we need to simulate qualitatively the system in order to propagate these deviations. The description of a system is made in three different layers (Kuipers, 1984):

1. Structural description: variables that characterize the system, such as flow $(\mathrm{F})$, pressure $(\mathrm{P})$, temperature $(\mathrm{T})$, composition $(\mathrm{x})$, energy $(\mathrm{E})$, information (I), level (L), valve opening (A), etc. The symbols in brackets will be used in D-higraphs.

2. Behavioral description: potential behavior of the system as a network. The $\mathrm{M}^{+}$and $\mathrm{M}^{-}$constraints (Kuipers, 1986) provide this information and they we will use the following compacted compacted notation:

$$
Z_{Y_{1}, Y_{2} \ldots Y_{m}}^{X_{1}, X_{2}, X_{n}} \Leftrightarrow M^{+}\left(X_{i}, Z\right) \wedge M^{-}\left(Y_{i}, Z\right) \quad \forall i, j
$$

3. Functional description: purpose of a structural component of connections; provided by the D-higraphs layout.

The three layers of this representation are shown in the right-hand-side of Fig. 1, where there is a physical device (DECANTER) whose main purpose is to store liquid. The decanter has two characteristic variables: level (Ld) and temperature (Td). Ld is affected by the inflow F3 with variations of the same sign (an increment of F3 increases Ld) and by the outflow F4 in the opposite way. In the same way, the flow F4 is affected by the level of the decanter in the same direction (see Eq. 1). 


\section{D-higraphs environment and HAZOP Assistant}

D-higraphs are developed using the environment shown in Fig. 2. The models are implemented using a graphic tool Álvarez (2010). This tool has as input the P\&ID of the process and it uses a D-higraphs built in template. Once the model has been developed, it is loaded into the expert system. The HAZOP study is performed feeding the deviations to the reasoning engine. The result of the analysis, a causal tree, is provided to the user and they can be fed back to the modeling tool in order to make changes into the process and/or D-higraph.

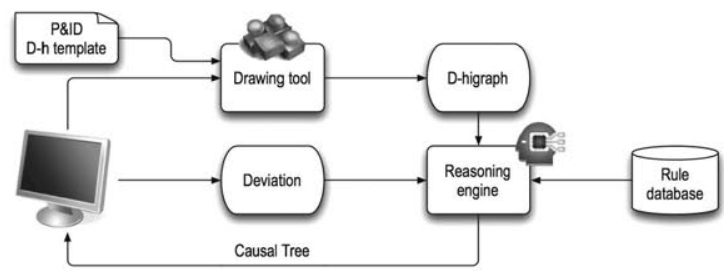

Fig. 2. D-higraphs environment.

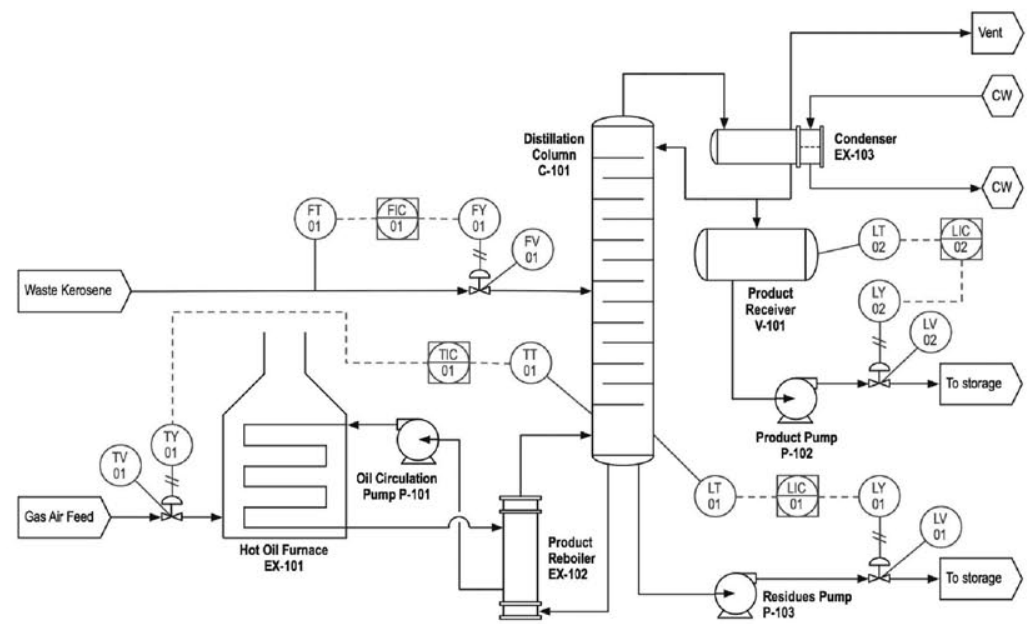

Fig. 3. Kerosene recovery unit.

\section{Kerosene recovery unit (case study)}

The kerosene recovery unit shown in Fig. 3 is part of a proposal of DOP Refineries Ltd. for the construction of a refinery for the recovery of kerosene from the waste kerosene solvent returned from auto engine repairers. This process and its conventional HAZOP analysis is taken from the "Hazardous Industry Planning Advisory Paper No. 8" (2011).

\subsection{Functional decomposition}

The main goal of the unit is to recover the kerosene from the waste kerosene solvent. To that end, the system can be decomposed into its subsystems, which perform the necessary subfunctions and subgoals, in the following way: (1) Feed section: provide a constant feed flow, (2) Distillation column: separate the kerosene, (3) Reflux section: provide reflux to the column and remove the recovered kerosene, and (4) Reboiler section: provide energy to the column. 
This decomposition can be continued until the desired level of detail. The D-higraph is developed using this decomposition and the P\&I diagram. However, in this paper we only show a part of the overall D-higraph due to space constraints. See Fig. 4 for the Dhigraph of the reboiler section.

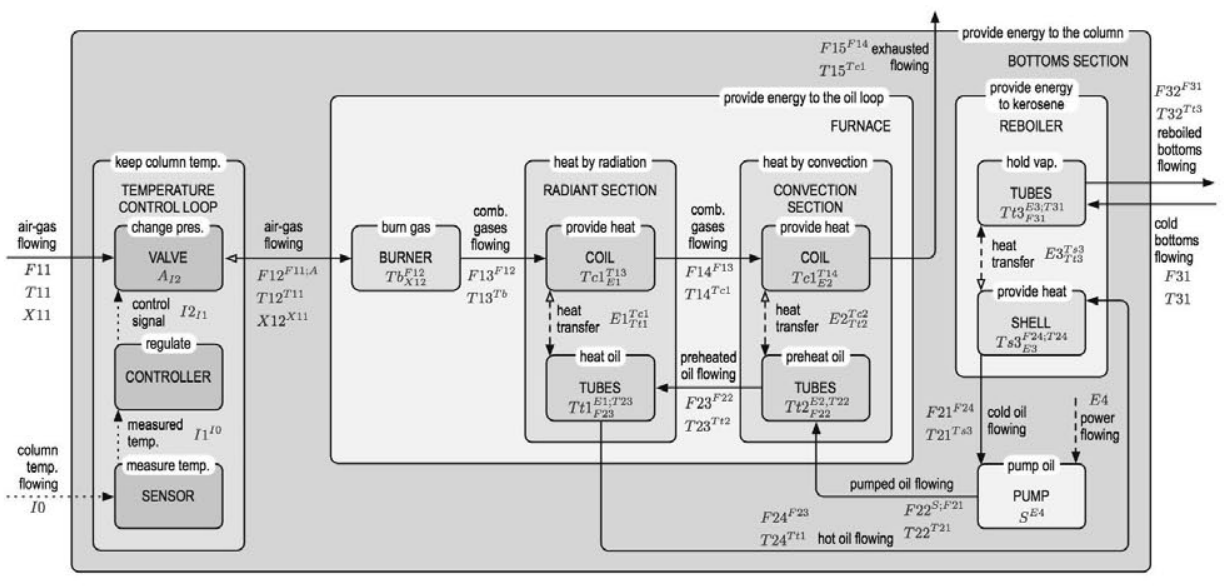

Fig. 4. Reboiler section D-higraph.

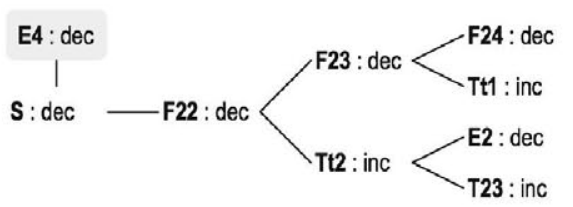

Deviation 1: Low energy to the oil pump.

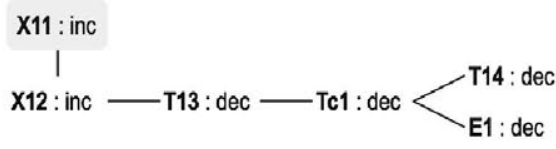

Deviation 2: Too much air in the air-gas feed.

Fig. 5. Consequences trees for the deviations considered.

\subsection{Deviation 1: Low energy to the oil pump.}

This deviation consists of the variable "power flowing" to the oil pump (E4) and the guide- word "less of". Its consequences tree (with depth 4) are show in Fig. 5. This tree can be expanded to cover all of the D-higraph. The conclusions that can be obtained are: (1) The temperature of the oil can be to high and end in its degradation, (2) the temperature of the reboiled flow would decrease, and (3) the temperature of the exhausted would increase, which means that we are wasting energy.

\subsection{Deviation 2: Too much air in the air-gas feed.}

This deviation consists of the variable "air-gas composition" (X11) and the guide-word "more of". The consequences tree for this deviation (with depth 4) are shown in Fig. 5. Again, this tree can be expanded to drawn these consequences: (1) low temperature of the oil in its loop, and (2) low temperature of the reboiled flow.

Of course, in both deviations, the trees can be expanded to cover all of the process, only the depth of the tree has to be changed. With this, the analysis can cover all of the plant and not only the node under consideration. 


\section{Comparison with other methodologies}

Conventional HAZOP studies are systematic and a logical way of performing PHA. However, $70 \%$ of the time they require is devoted to routine deviations (Venkatasubramanian et al., 2000). The automation of the procedure saves time and hence money. Automating HAZOP, the team can spend their time in analyzing the deviations, causes, consequences and the possible solutions and not in obtaining them. Another advantage of automated HAZOPs is that no nodes are left unexplored. The proposed assistant only needs a model (a D-higraph) of the process and not a model for each node of the HAZOP analysis, like the MFM HAZOP assistant (Rossing et al., 2010). The rule database is also common for all of the process because it is related to the relations between the elements of the model and not to the process itself, like HAZOPExpert (Venkatasubramanian et al., 2000), however, HAZOPExpert is able to deal with batch processes. Another advantage of this approach, when compared with the MFM methodology, is that the conclusions of the study can be directly mapped to the devices and equipment of the process. This is a consequence of the functional and structural integration.

\section{Conclusions and further work}

We have presented a tool that allows us to perform guided HAZOP studies. This tool uses a functional model, D-higraph, that merges functional and structural information about the process under analysis. This tool has been applied to an industrial case to show its applicability and to compare it with other existing tools. The resulting analyses are more complete, easier to perform and directly related to the process due to the integration of functions and structures. Future work will be devoted to the development of a "translator" between P\&IDs and D-higraphs, so the models can be obtained from the diagrams by adding additional information. Further work will also include the integration of this tool with quantitative information and models.

\section{References}

Álvarez, M. E. 2010. Diagnosis de fallos en procesos químicos mediante modelos D-higraph. Final Project. Department of Chemical Engineering, Technical University of Madrid.

CLIPS. 2011. CLIPS, A Tool for Building Expert Systems. http://clipsrules.sourceforge.net

De la Mata, J. L., Rodríguez, M. 2010a. Abnormal situation diagnosis using D-higraphs. Proc. of the $20^{\text {th }}$ European Symposium on Computer Aided Process Engineering, pp. 1477-1482.

De la Mata, J. L., Rodríguez, M. 2010b. D-higraphs. ASLab Report. http://www.aslab.org

Department of Planning, State of New South Wales, Australia. 2011. Hazardous Industry Planning Advisory Paper No. 8. HAZOP Guidelines.

Harel, D. 1987. Statecharts: A visual formalism for complex systems. Sci. Comput. Program., 8.

Kuipers, B. 1984. Commonsense reasoning about causality. Artificial Intelligence, 24.

Kuipers, B. 1986. Qualitative simulation. Artifiial Intelligence, 29.

Lind, M. 1994. Modeling goals and functions of complex Industrial plant. Applied Artificial Intelligence, 8 (2).

Lind, M. 2005. Modeling goals and functions of control and safety systems. NKS Report.

Rodríguez, M., Sanz, R. 2009. Development of integrated functional-structural models. Computer Aided Process Engineering, 27.

Rossing, N. L., Lind, M., Jensen, N., Jorgensen, S. B. 2010. A functional HAZOP methodology. Computers and Chemical Engineering, 34 (2).

Venkatasubramanian, V., Zhao, C., Viswanathan, S. 2000. Intelligent systems for HAZOP analysis of complex process plants. Computers and Chemical Engineering, 24 (9-10).

Zhao, C., Bhushan, M, \& Venkatasubramanian, V. 2005. PHASuite: An Automated HAZOP Analysis Tool for Chemical Processes. Process Safety and Environment Protection, 83 (6). 